\title{
Evaluation of the crystallographic fatigue crack growth rate in a single-crystal nickel-base superalloy
}

Christian Busse, Frans Palmert, B. Sjodin, P. Almroth, D. Gustafsson, Kjell Simonsson and Daniel Leidermark

The self-archived postprint version of this journal article is available at Linköping University Institutional Repository (DiVA):

http://urn.kb.se/resolve?urn=urn:nbn:se:liu:diva-160388

N.B.: When citing this work, cite the original publication.

Busse, C., Palmert, F., Sjodin, B., Almroth, P., Gustafsson, D., Simonsson, K., Leidermark, D., (2019), Evaluation of the crystallographic fatigue crack growth rate in a single-crystal nickel-base superalloy, International Journal of Fatigue, 127, 259-267. https://doi.org/10.1016/j.ijfatigue.2019.05.023

Original publication available at:

https://doi.org/10.1016/j.ijfatigue.2019.05.023

Copyright: Elsevier

http://www.elsevier.com/

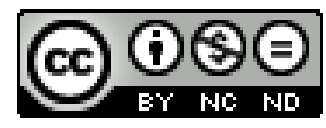




\title{
Evaluation of the crystallographic fatigue crack growth rate in a single-crystal nickel-base superalloy
}

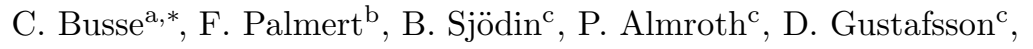 \\ K. Simonsson ${ }^{\mathrm{a}}$, D. Leidermark ${ }^{\mathrm{a}}$ \\ ${ }^{a}$ Division of Solid Mechanics, Linköping University, SE-58183 Linköping, Sweden \\ ${ }^{b}$ Division of Engineering Materials, Linköping University, SE-58183 Linköping, Sweden \\ ${ }^{c}$ Siemens Industrial Turbomachinery AB, SE-61283 Finspång, Sweden
}

\begin{abstract}
Cracks in single-crystal nickel-base superalloys have been observed to switch cracking mode from Mode I to crystallographic cracking. The crack propagation rate is usually higher on the crystallographic planes compared to Mode I, which is important to account for in crack growth life predictions. In this paper, a method to evaluate the crystallographic fatigue crack growth rate, based on a previously developed crystallographic crack driving force parameter, is presented. The crystallographic crack growth rate was determined by evaluating heat tints on the fracture surfaces of the test specimens from the experiments. Complicated crack geometries including multiple crystallographic crack fronts were modelled in a three dimensional finite element context. The data points of the crystallographic fatigue crack growth rate collapse on a narrow scatter band for the crystallographic cracks indicating a correlation with the previously developed crystallographic crack driving force.
\end{abstract}

Keywords: Single-crystal nickel-base superalloys, Finite element analysis, Fracture mechanics, Stress intensity factor, Crystallographic cracking, Fatigue crack growth rate

\footnotetext{
${ }^{*}$ Corresponding author, Tel.: +46 700896003

Email address: christian.busse@liu.se (C. Busse)
}

Preprint submitted to International Journal of Fatigue

May 9, 2019 


\section{Introduction}

There is a continuous strive towards higher firing temperatures when designing industrial gas turbines used for power generation, due to the resulting increase in efficiency and reduction of the carbon footprint [1]. A consequence of the increasing temperatures are higher requirements on the materials used in components in the hot sections of the gas turbines. Essential components that are exposed to this severe environment are e.g. the gas turbine blades. These blades are therefore often manufactured from nickel-base superalloys cast as single-crystals, due to their superior material properties under these working conditions. However, the complex nature of the anisotropy in these materials is often challenging in terms of testing, modelling and understanding the fracture behaviour [2-8]. The crystallographic orientation of single-crystal materials has been shown to influence the crack growth behaviour [9-11], and is thus an important factor in the modelling. Growing cracks have been observed to, under certain conditions, change cracking mode from Mode I to cracking on crystallographic slip planes [11-16], see Fig. 1 where both cracking modes can be seen. Crystallographic cracking differs from Mode I cracking in its mechanisms, resulting in a higher Fatigue Crack Growth Rate (FCGR) [17], and it is thus important to have a methodology that enables a reliable prediction. The Mode I crack growth behaviour is well understood and easier to model compared to the crystallographic cracking mode. One major aspect complicating the modelling of the behaviour of single-crystal materials is the dependence on the crystallographic orientation including the misalignments, influencing the stress state and thus the fracture behaviour $[18,19]$. Especially Three-Dimensional (3D) crack growth modelling incorporating crystallographic cracking is of great complex-

ity due to the complicated crack geometry where multiple crack fronts can be present.

Previous research concerning crystallographic FCGRs has been restricted to Two-Dimensional (2D) evaluations $[8,12,15]$ or simple crack geometries with only one crystallographic crack in $3 \mathrm{D}[9,11]$. Several fracture mechanics 


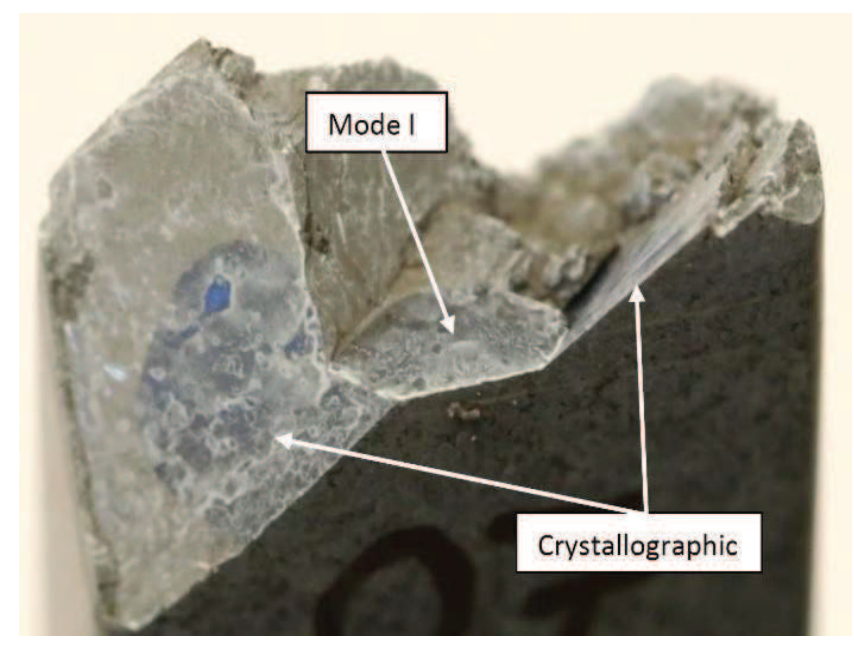

Figure 1: Fracture surface of test specimen Kb1 showing two distinct cracking modes.

approaches for single-crystal superalloys are available in the literature, where most of the published literature deemed Resolved Shear Stresses (RSSes) to be an appropriate entity for applications with single-crystal materials where crystallographic cracking might occur [14, 20, 21]. A different approach concerning the same topic was presented Sakaguchi et al. [19] using crystal plasticity to evaluate crystallographic crack growth.

The aim of this work is to develop a methodology to evaluate the FCGRs for crystallographic cracking applicable to arbitrary crack geometries. This has been done by numerically evaluating appropriate Crack Driving Force (CDF) parameters along the crystallographic crack fronts by the use of the Finite Element (FE) method and correlating it to a crack growth model. The model has been calibrated by isothermal low-cycle fatigue experiments at $500^{\circ} \mathrm{C}$. This has been done by assessing heat-tinted fracture surfaces from the performed experiments, where the crack front shapes and the corresponding number of elapsed cycles are known. Furthermore, Mode I FCGRs are presented for the sake of comparison. The Mode I FCGRs are based on the evaluations of the performed experiments in this study, and on a different specimen geometry [22].

The presented methodology is applicable to arbitrary 3D FE-models and 
crystallographic orientations. This aspect is of high importance for industrial applications, where a more accurate prediction of the FCGR in single-crystal components can increase maintenance intervals as well as the efficiency of the design. The authors are not aware of similar research using 3D calculations for arbitrary crack geometries and crystallographic orientations.

\section{Material and Experiments}

The investigated material is a single-crystal nickel-base superalloy of facecentred cubic structure, similar to the alloy described by Reed et al. [23]. Its main alloying elements, in order of decreasing wt \%, are as follows: Ni-Cr-TaCo-Al-W-Mo-Si-Hf-C-Ce.

Since the specimens were cast as only one single grain they possess anisotropic properties, where the elastic stiffness constants for $500^{\circ} \mathrm{C}$ are presented in Table 1 . The temperature of $500^{\circ} \mathrm{C}$ has been chosen since it represents the temperature during normal operation at the blade fir tree root, which is a critical location in the blade design.

Table 1: Elastic stiffness constants, units in GPa.

\begin{tabular}{llll}
\hline Temp. $\left({ }^{\circ} \mathrm{C}\right)$ & $\mathrm{C}_{11}$ & $\mathrm{C}_{12}$ & $\mathrm{C}_{44}$ \\
\hline 500 & 201.9 & 127.0 & 112.8 \\
\hline
\end{tabular}

Three isothermal fatigue crack growth experiments at $500^{\circ} \mathrm{C}$ were performed. The test specimen geometry was a surface flawed fatigue crack growth specimen of Kb-type [24], henceforth denoted as Kb specimen. The geometry of the Kb specimen can be seen in Fig. 2 .

The specimens were manufactured from cast bars with the casting direction parallel to the nominal [001] crystallographic direction. The nominal secondary orientation [010] was normal to the wide surface in the centre of the specimen on which also the flaw was introduced, $c f$. Fig. 2. The fatigue crack growth experiments were performed according to the test conditions shown in Table 2. Further details of the experimental procedure can be found in [25]. Note 

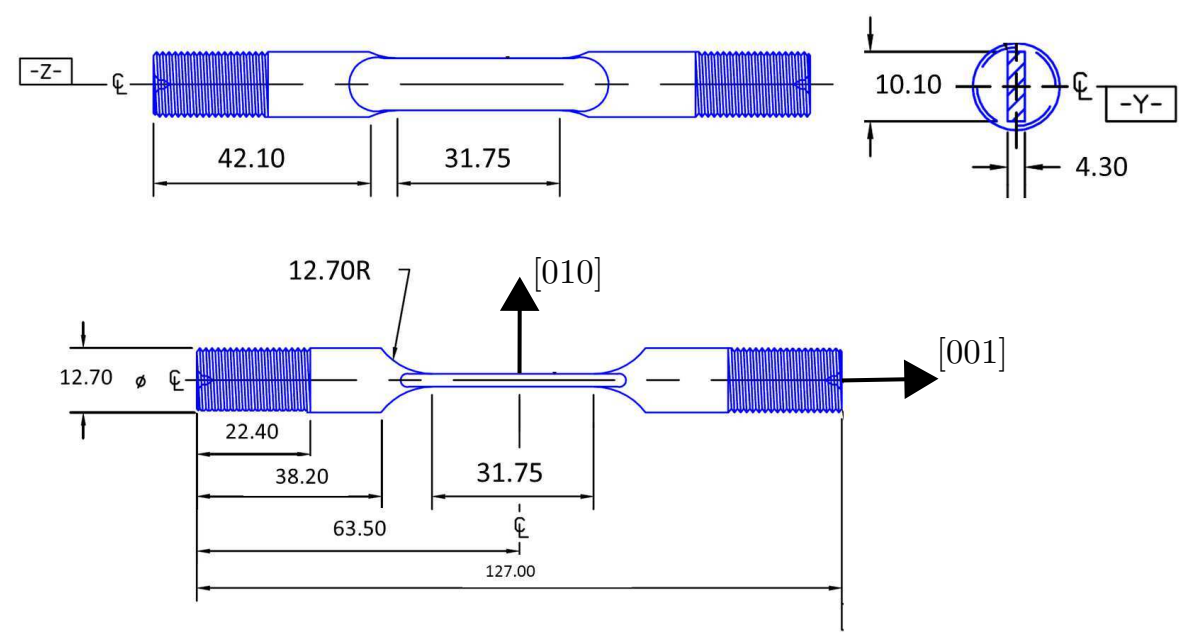

Figure 2: Technical drawing of the Kb specimen with the nominal primary and secondary orientations marked. Units in mm.

that the specimens used in experiment $\mathrm{Kb} 2$ and $\mathrm{Kb} 3$ in this work are different than $\mathrm{Kb} 2$ and $\mathrm{Kb} 3$ in [25], where they were not included. However, the same notation sequence was used here.

Table 2: Overview of the test conditions in the experiments of the Kb specimens.

\begin{tabular}{llll}
\hline Specimen & Kb1 & Kb2 & Kb3 \\
\hline Temperature $\left[{ }^{\circ} \mathrm{C}\right]$ & 500 & 500 & 500 \\
\hline Stress ratio $R_{\sigma}[-]$ & 0.05 & 0.05 & 0.05 \\
\hline Frequency $[\mathrm{Hz}]$ & 0.5 & 10 & 0.5 \\
\hline Max. Force $[\mathrm{N}]$ & 19857 & 18407 & 27285 \\
\hline Loading direction & {$[001]$} & {$[001]$} & {$[001]$} \\
\hline
\end{tabular}

The misalignments from the nominal crystallographic orientations in terms of sequential rotations about the nominal [010], [100] and [001] directions are shown in Table 3 and were determined by measuring the visible dendrites representing the projections of the crystallographic lattice vectors on three polished and etched orthogonal surfaces on the specimens. For a detailed description 
see [25].

During the experiments the projected cracked area was monitored using direct current Potential Drop (PD) measurement. The PD signal was used to approximate the crystallographic crack lengths as discussed below. Furthermore, three heat tints were created in each test by applying a hold-time under tension to ensure an open crack. The resulting oxidation on the crack surfaces represents the heat tints, i.e. the crack front shape at that moment. This has been done at known number of cycles so that the heat tints are approximately equally spaced on the crack surface to generate a useful crack length versus PD signal curve. In the following, the cracks associated with the corresponding heat tints and also the the approximated crack shape at cracking mode transition will be denoted as crack instances, i.e. there are four crack instances for Kb1 and $\mathrm{Kb} 2$ and three crack instances for Kb3. The lower number of crack instances in $\mathrm{Kb} 3$ is due to that the cracking mode transition occurs directly from the Electro Discharge Machined (EDM) notch and thus the crack instance associated to the cracking mode transition has been omitted.

Table 3: Mean crystallographic orientation accounting for the misalignment in terms of sequential rotation about the nominal [010], [100] and [001] directions.

\begin{tabular}{lrrr}
\hline Specimen & Kb1 & Kb2 & Kb3 \\
\hline Rotation about $[010]\left[^{\circ}\right]$ & 1.7 & 0.3 & 0.3 \\
\hline Rotation about $[100]\left[^{\circ}\right]$ & 0.1 & 2.2 & -1.2 \\
\hline Rotation about $[001]\left[^{\circ}\right]$ & -2.8 & 3.4 & 0.9 \\
\hline
\end{tabular}




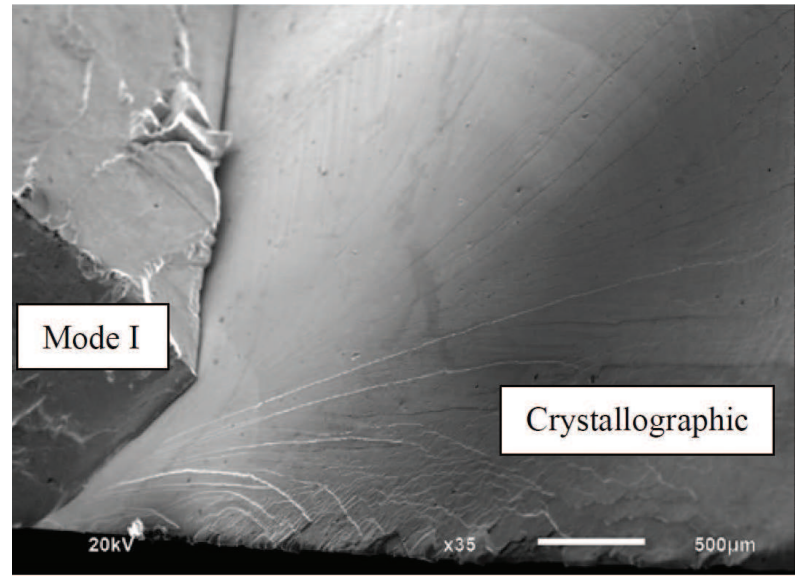

(a)

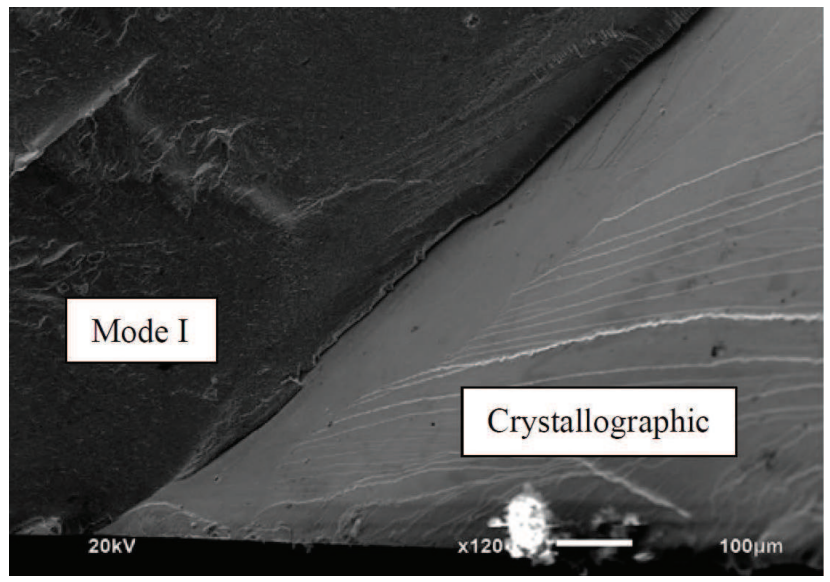

(b)

Figure 3: SEM image of the transition of cracking modes, showing (a) the crystallographic crack front advancing beneath the Mode I crack front and (b) in higher magnification.

After the experiments were finished, the fracture surfaces of all specimens were examined. As an example, the fracture surface of the specimen Kb1 with its cracking mode transitions from Mode I to crystallographic cracking on each side can be seen in Fig. 1. Further examination in a Scanning Electron Microscope (SEM) at the location where the two cracking modes intersect was performed. This is depicted in Fig. 3 for Kb1, where the cracking mode transition on the right side of the Mode I crack is shown. It can be observed that the progressing crystallographic crack front advanced on a crystallographic plane beneath the Mode I crack front. At higher magnification, $c f$. Fig. 3(b), it can be seen that the crystallographic crack grew beneath the Mode I crack all the way from the free surface. A similar behaviour was observed for the specimen Kb2.

However, the crack in the specimen Kb3 transitioned directly to crystallographic cracking on three different crystallographic planes from the EDM notch, see Fig. 4. This means that no Mode I crack (besides the EDM notch) is present. It can be seen that the crystallographic crack fronts are continuous for all crack instances. Even though there is only one crack with a continuous front, in the context of this work each portion of the crack which is on a different crystallo- 
graphic plane is regarded as a separate crack. It should be noted that one of these cracks in fact grew on two crystallographic planes which have the same orientation and are parallel to each other. The location of the intersection is highlighted by the red arrow in Fig. 4. For the sake of simplicity, within an engineering context, they were considered as one crystallographic plane in the further evaluations.

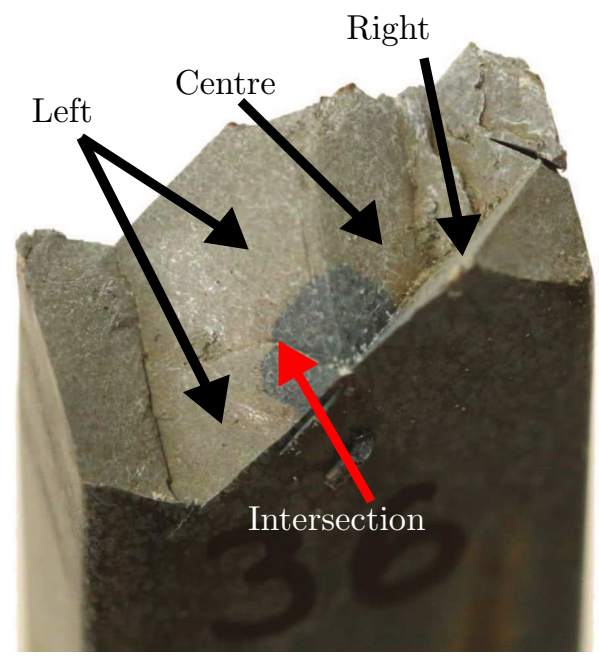

Figure 4: Fracture surface of test specimen Kb3. The intersection between two parallel crystallographic planes is highlighted by the red arrow. Definition of the left, centre and right crystallographic cracking plane.

Additionally, it can be said that the Mode I crack growth might in reality consist of crystallographic crack growth on two conjugate slip planes on a microscopic level [7]. However, SEM investigations in a different study [25] on the same material indicated that the crack has grown between the $\gamma^{\prime}$-particles and thus, considering the context of this work, which is from an engineering point of view, the cracking mode which is perpendicular to the loading direction is macroscopically regarded as a Mode I crack and treated separately from the crystallographic cracks.

Furthermore, the fracture surfaces have been studied in an optical microscope in order to evaluate the crack front shapes for the different heat tints. 
The first, second and third heat tints for Kb1 are shown for the crystallographic and the Mode I crack fronts in Fig. 5, where they are highlighted as solid lines in black, green and red. The crack front shape at cracking mode transition is highlighted in blue and approximated to be of semi-circular appearance. The crack shapes of the hidden portions of the two crystallographic crack fronts (on the left and on the right side of the Mode I crack front) have been approximated and are represented by the dashed lines in Fig. 5. In the following, the crack instances outlined by the black, blue, green and red contours associated to the crack shape at the first heat tint, at the cracking mode transition, and at the second and third heat tints are referred to as crack instance 0, 1,2 and 3 , respectively. Crack instance 0 corresponds to the heat tint of a pure Mode I crack in $\mathrm{Kb} 1$ and $\mathrm{Kb} 2$. As there is no transition in Kb3 no crack instance 1 is present. From the analysed fracture surfaces it can be concluded that, for Kb1, crack instances 2 and 3 include three intersecting crack fronts, i.e. two crystallographic fronts and one Mode I front, $c f$. Fig. 5. For Kb2, only one occurrence of crystallographic cracking is present for crack instance 2, which is the one on the left side of the Mode I crack, see Fig. 6. For Kb3 all crack instances contain the three crystallographic cracks as shown in Fig. 7. Comparing the areas between the black solid lines (heat tint 1) and the green solid lines (heat tint 2) for Kb1 and Kb2 in Figs. 5 and 6, it can be seen that they are of similar appearance. This is not the case for Kb3 in Fig. 7, where the area is different. This can be explained by the irregular crack front shape indicated by the heat tints in Kb3. Further, the visible crystallographic cracks are inclined in three dimensions but only the 2D projection of the fracture surface is visible in Fig. 7. This might distort the sense of how the image is perceived. The cracks in Kb1 and $\mathrm{Kb} 2$ grew more regular as semi-circular cracks on the Mode I plane.

Each heat tint and the cracking mode transition is associated to a number of loading cycles until that specific crack shape is formed. The number of loading cycles are presented in Table 4 , where $N_{0}, N_{1}, N_{2}$ and $N_{3}$ represent the number of cycles until crack instance 0, 1 (transition), 2 and 3 was reached. The number of elapsed cycles until crack instance 1 was derived from the PD 


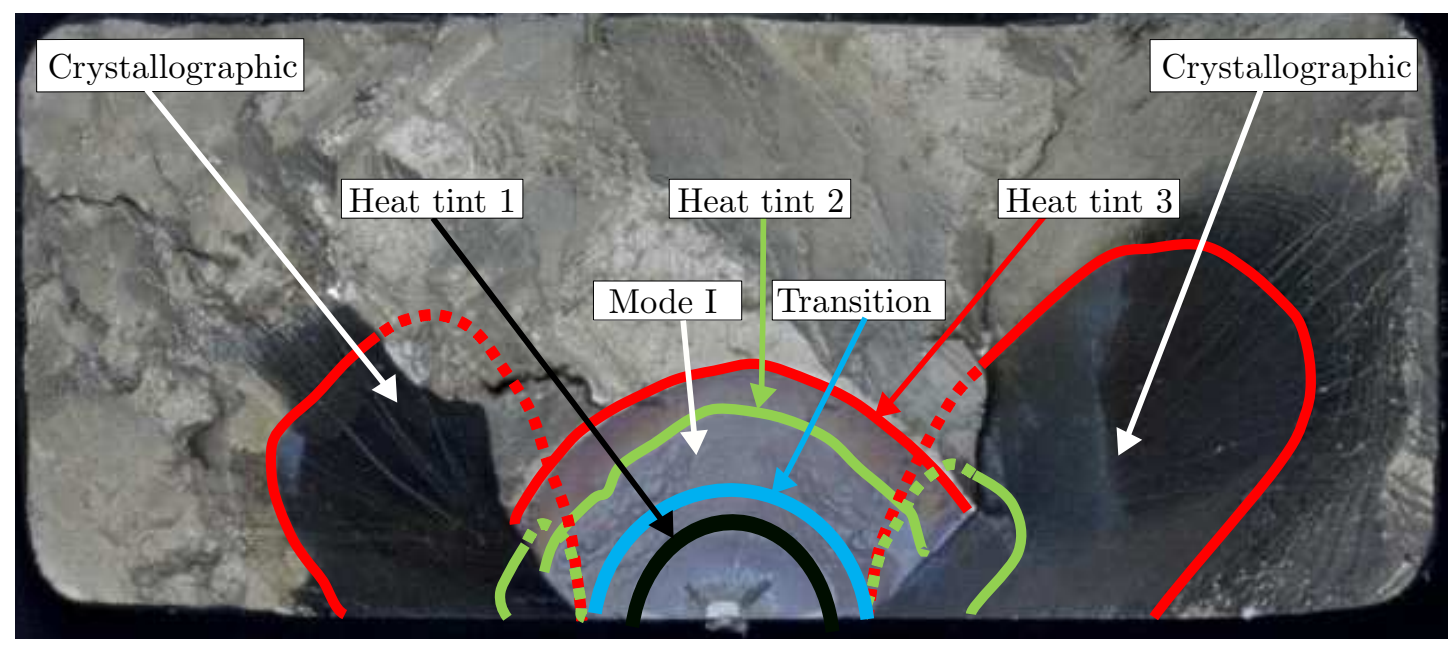

Figure 5: Highlighted heat tints on the fracture surface of specimen Kb1. Cracks instances $0,1,2$ and 3 are highlighted in black, blue, green and red, respectively. Note that this is the opposite fracture surface of the cracked specimen compared to what is shown in Fig. 1.

signal, which enables a reliable evaluation for the assumed semi-circular Mode I crack appearance until the cracking mode transition. It should be noted that a straight-forward determination of the crack length by use of the PD signal was not possible once crystallographic cracking occurred. This is due to the irregularity of the crack path. Nevertheless, after the cracking mode transition, the PD signal has been used to approximate the crystallographic crack length as a function of elapsed cycles, which is further discussed below.

Table 4: Experimental results of the number of cycles for the different heat tints.

\begin{tabular}{|c|c|c|c|c|}
\hline Specimen $\quad$ Crack instance & $N_{0}$ & $N_{1}$ (transition) & $N_{2}$ & $N_{3}$ \\
\hline Kb1 & 203678 & 209765 & 217426 & 218827 \\
\hline $\mathrm{Kb} 2$ & 1010 & 15803 & 29249 & 34492 \\
\hline Kb3 & 256 & - & 2153 & 2587 \\
\hline
\end{tabular}

Furthermore, the fracture surfaces were scanned in an Atos Triple Scan machine to acquire a $3 \mathrm{D}$ representation of the fracture surfaces. This data, in 


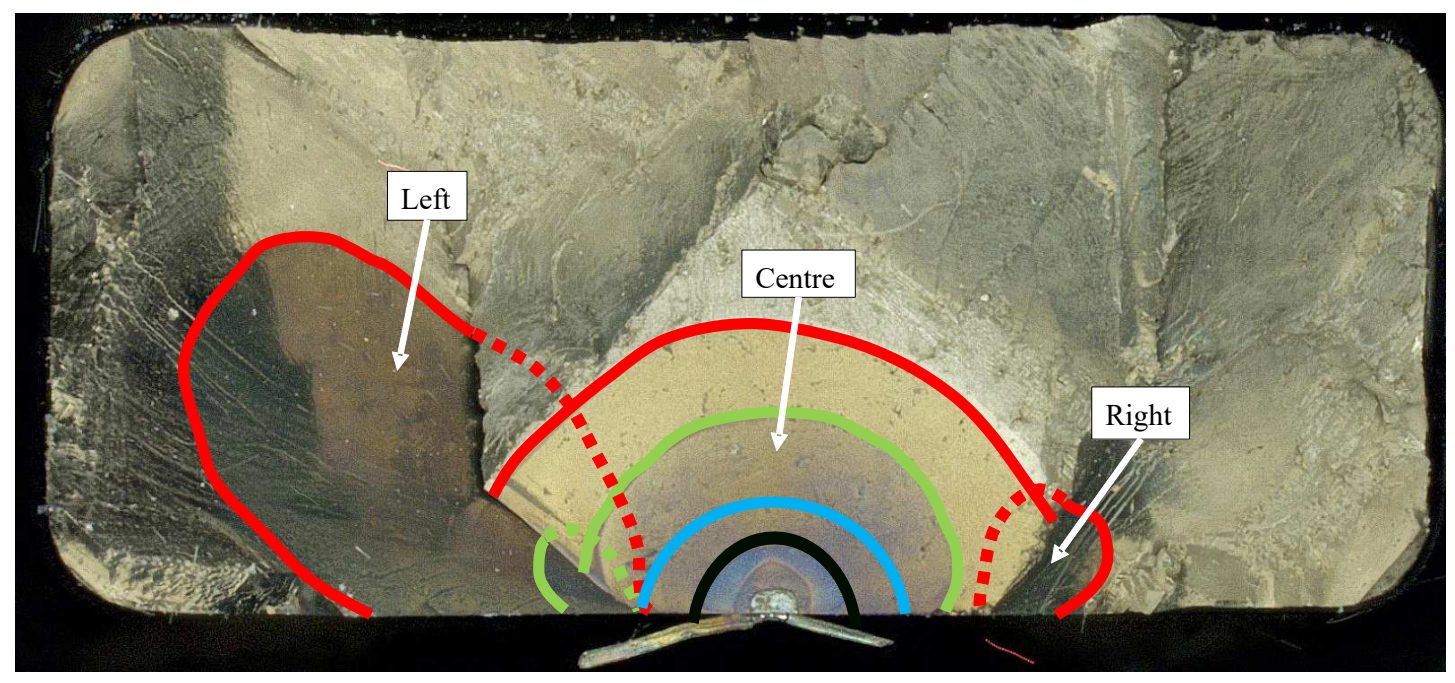

Figure 6: Heat tints on fracture surface of specimen Kb2. Cracks instances 0, 1, 2 and 3 are highlighted in black, blue, green and red, respectively. Illustration of the crack front positions left, centre and right.

conjunction with the optical images were used to create a realistic mapping of the crack surface geometry into a 3D triangulation used in FE modelling of the experiments. The 3D representations of the fracture surfaces were also used to identify the cracking plane normals for all crack fronts in all crack instances. The active cracking planes, in terms of Miller notation, for the left, centre and right side are summarized in Table 5. The cracking planes of Kb1 and Kb2 follow the definitions in Fig. 6. The corresponding definition of the cracking planes for Kb3 can be seen in Fig. 4. 


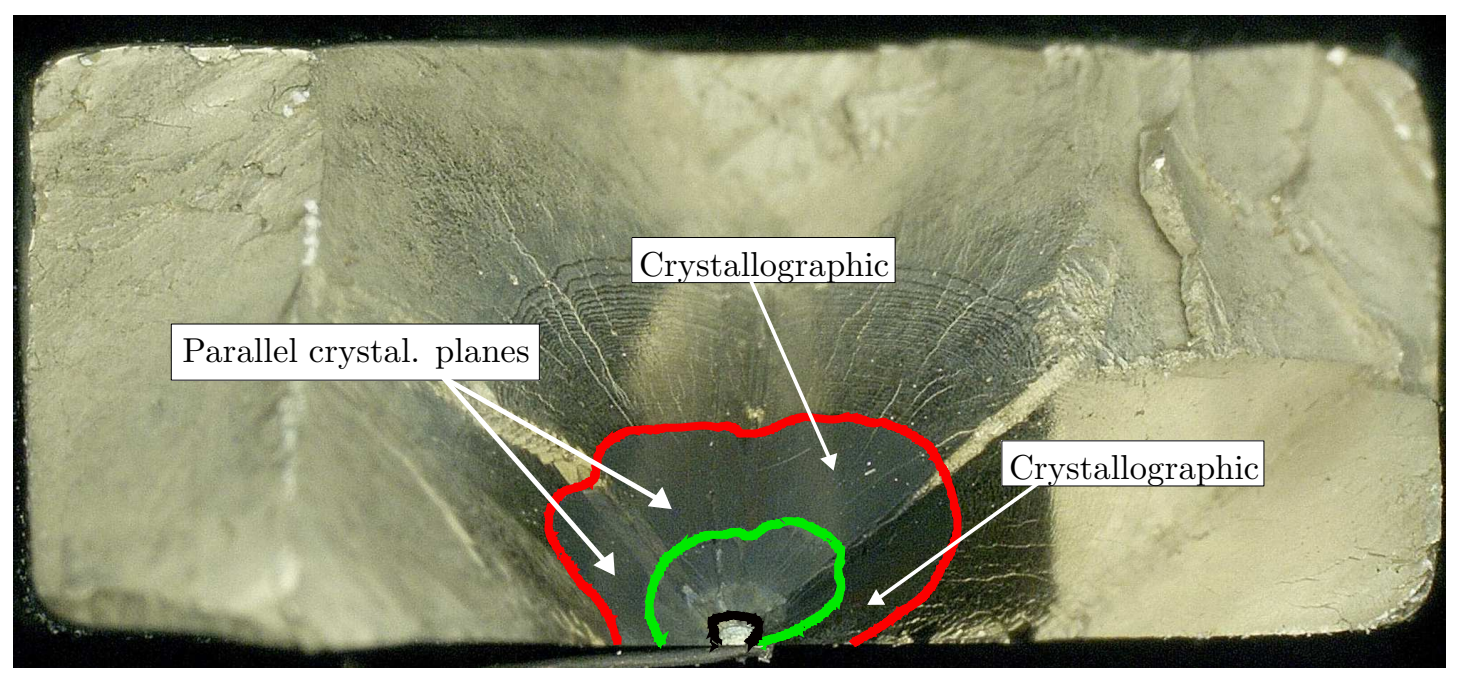

Figure 7: Heat tints on fracture surface of specimen Kb3. Cracks instances 0, 2 and 3 are highlighted in black, green and red, respectively. The two parallel crystallographic planes are marked.

Table 5: Normals of the observed cracking planes in Miller notation for the left, centre and right side seen from the crack initiation point for all three crack instances and test specimens.

\begin{tabular}{c|ccc|ccc|ccc} 
& \multicolumn{3}{|c|}{ Crack Instance 0/1* } & \multicolumn{3}{c|}{ Crack Instance 2 } & \multicolumn{3}{|c}{ Crack Instance 3 } \\
\hline & Left & Centre & Right & Left & Centre & Right & Left & Centre & Right \\
\hline Kb1 & - & {$[001]$} & - & {$[\overline{1} 1 \overline{1}]$} & {$[001]$} & {$[\overline{1} \overline{1} 1]$} & {$[\overline{1} 1 \overline{1}]$} & {$[001]$} & {$[\overline{1} \overline{1} 1]$} \\
\hline Kb2 & - & {$[001]$} & - & {$[1 \overline{1} \overline{1}]$} & {$[001]$} & - & {$[1 \overline{1} \overline{1}]$} & {$[001]$} & {$[111]$} \\
\hline Kb3 & {$[\overline{1} 1 \overline{1}]$} & {$[111]$} & {$[\overline{1} \overline{1} 1]$} & {$[\overline{1} 1 \overline{1}]$} & {$[111]$} & {$[\overline{1} \overline{1} 1]$} & {$[\overline{1} 1 \overline{1}]$} & {$[111]$} & {$[\overline{1} \overline{1} 1]$} \\
\hline
\end{tabular}

${ }^{*}$ Crack instance 1 not applicable for Kb3 


\section{Crystallographic Fatigue Crack Growth Rate}

In order to evaluate the crystallographic FCGR in a crack growth model, an appropriate CDF parameter has to be adopted. The correct choice of CDF parameter is vital for a meaningful prediction of the crystallographic crack growth rate. The equivalent Resolved Stress Intensity Factor (RSIF) $k_{E Q}$ used in this work for the crystallographic portions of the crack has been adopted from Busse et al. [25] and describes the singular stress field in the crack vicinity resolved onto the crystallographic slip planes in the Burgers' directions. This is to account for the fact that the deformations in these materials are localised to the crystallographic slip planes in the Burgers' directions, and are associated to the dislocation motion [26]. As there are three Burgers' direction per crystallographic slip plane, the direction yielding the highest $k_{E Q}$ value is chosen for the evaluated point at the crack fronts in the further crack growth model evaluation. The equivalent RSIF $k_{E Q}$ is based on anisotropic Stress Intensity Factors (SIFs) $\left(K_{I}, K_{I I}\right.$ and $\left.K_{I I I}\right)$, which can be evaluated in different ways. The approach in this work has been by using the M-Integral [27] with incorporation of anisotropic material properties and accounting for the crystallographic orientations including the material misalignments. The RSIFs describe the three modes of fracture resolved on the crystallographic planes in the Burgers' directions. This is illustrated in Fig. 8 and the equivalent RSIF parameter $k_{E Q}$ is defined as:

$$
k_{E Q}=\sqrt{\psi k_{I}^{2}+k_{I I}^{2}+k_{I I I}^{2}}
$$

The Mode I RSIF is scaled with $\psi$, which is a calibration parameter based on experiments in order to predict the correct crystallographic cracking planes along a Mode I crack front. For more in-depth details see [25].

The above presented equivalent RSIF parameter is employed in a power law crack growth model, as proposed by Paris et al. [28], according to:

$$
\left(\frac{d L}{d N}\right)_{c}=C_{c}\left(\Delta k_{E Q}\right)^{n_{c}}
$$

which represents the crack growth for crystallographic cracking with the pa- 


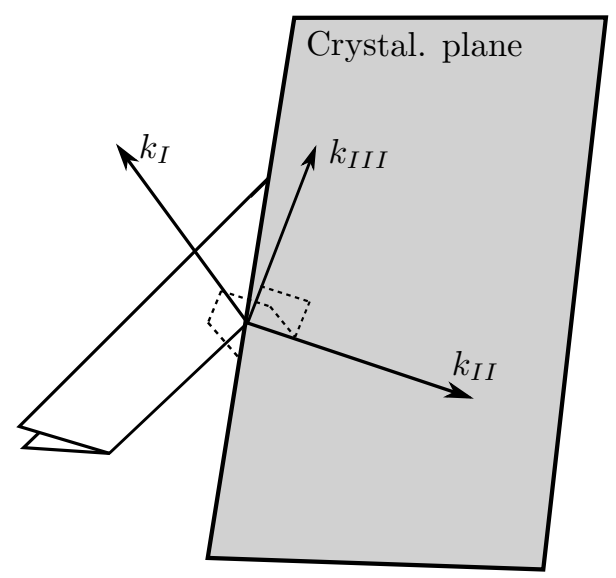

Figure 8: Illustration of the directions of the RSIFs on a crystallographic plane.

rameters $C_{c}$ and $n_{c}$, where the subscript $c$ indicates that the parameters refer to crystallographic cracking and $(d L / d N)_{c}$ is the crystallographic FCGR. The RSIF range was taken to be $\Delta k_{E Q}=0.95 \cdot k_{E Q_{\max }}$ for a stress ratio of $R_{\sigma}=0.05$.

The crystallographic crack length $L$ is taken to be the maximum distance from the transition point, which can be identified by $L_{1}$ in Fig. 9, to the crack instances 2 and 3. The depicted transition point was chosen under the assumption that the transition to crystallographic cracking occurred first close to the free surface of the specimen. Since $L_{1}$ lies at the transition point, the crystallographic crack length is zero, i.e. $L_{1}=0$. This is also illustrated in Fig. 9 by the white arrow along which the crystallographic crack length is evaluated. Note that $L$ is defined on the crystallographic plane in three dimensions, whereas Fig. 9 shows the projection on the 2D plane. A different crack growth variable $(d L / d N)_{c}$ was chosen in order to distinguish the crystallographic crack growth from the Mode I crack growth, typically denoted by $d a / d N$. Hence, the use of $L$ in the evaluations of the crystallographic crack lengths compared to $a$.

In order to determine the crystallographic crack growth rate $(d L / d N)_{c}$, the heat tints and the above mentioned PD measurements have been used. The PD signal is related to the projected cracked area, which can be correlated with 


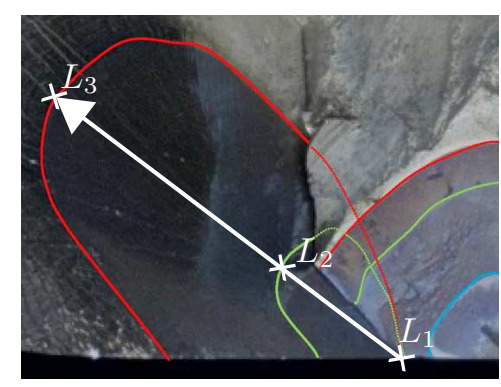

Figure 9: Representation of the crystallographic crack length $L$ in Kb1. The three instances, where the exact crack lengths are known are highlighted at $L_{1}, L_{2}$ and $L_{3}$.

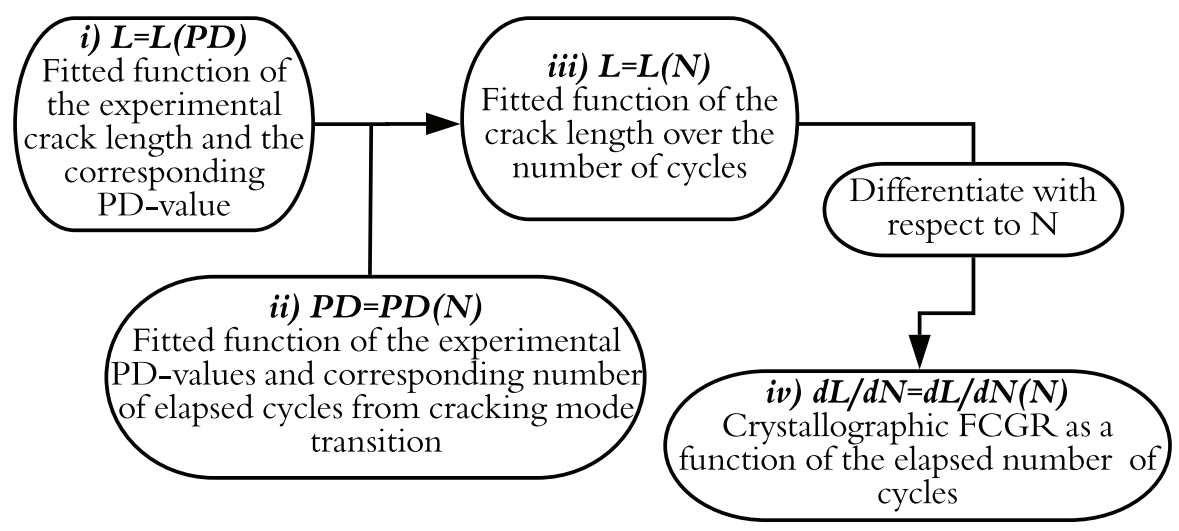

Figure 10: Flowchart describing the procedure to evaluate the crystallographic FCGR.

the crystallographic crack length using the definition of $L$ above. This was done in four steps, illustrated in Fig. 10;i) the crystallographic crack length can be expressed as a function of the PD signal. This was done for each crystallographic crack separately. The area of the crystallographic cracks and the crystallographic crack lengths were best approximated at the instances of the heat tints. Thus, for each visible heat tint, and also for the estimated transition crack shape, the corresponding crack length and PD signal were evaluated. This is exemplified in Fig. 9 for the left crystallographic crack of Kb1 with the three crystallographic crack lengths $L_{1}, L_{2}$ and $L_{3}$, which were used in the fitting; ii) the number of cycles $N$ and the PD signal were taken to start from zero at 
the transition to crystallographic cracking and fitted in a function; iii) the two above fitted functions were then combined to evaluate the crystallographic crack length $L$ as a function of $N$; and $i v$ ) derivated with respect to $N$ which gave the crystallographic FCGR $(d L / d N)_{c}$. A similar procedure was used to evaluate the RSIFs as functions of $N$. These relations were then used to calibrate the parameters $C_{c}$ and $n_{c}$ by fitting the crack growth data in a log-log diagram of the crystallographic FCGR over the RSIF parameter as described by Eq. 2.

The same procedure has been used to evaluate the Mode I FCGR $d a / d N$ using the Mode I SIF $K_{I}$ according to

$$
\frac{d a}{d N}=C_{I}\left(\Delta K_{I}\right)^{n_{I}}
$$

where the anisotropic SIF $K_{I}$ was computed using the M-Integral, as mentioned above.

\section{Simulations and evaluations}

In order determine the CDFs for all crack instances an FE-model of the Kb specimen was built. The following boundary conditions were applied; a fixed support at one of the circular surfaces and a concentrated force to one master node on the other circular surface. This force corresponds to the one in the experiments. The master node was coupled to all other nodes of the surface by a constraint that restrained motion in the plane and gave an equal deformation in the axial direction. The equivalent RSIF was then calculated along the crystallographic crack fronts. This was done for the cracks corresponding to the above defined crack instances. The anisotropic SIFs were calculated by the crack evaluation tool FRANC3D V7.3 [29] in conjunction with the FE-solver of ABAQUS V6.12 [30]. All simulations were performed in a linear elastic context using the elastic constants and misalignments given in Table 1 and 3 . The initial FE-model was divided into a global and a local domain. The crack geometry corresponding to the experiments was inserted in the local domain and then remeshed while the global domain retained its initial mesh. The two domains 
were then merged and analysed in the FE-solver, see Fig. 11. The resulting stress state was sent back to the crack evaluation tool, which calculated the anisotropic SIFs at each node along the crack fronts. In order to handle the anisotropic constitutive behaviour an elastic anisotropic material description was used. The crystallographic orientations including misalignments, $c f$. Table 3 , corresponding to the specimens in the experiments were accounted for in the material description. A more detailed description about the element types and the mesh configuration at the crack fronts in the FE-model can be found in [18].

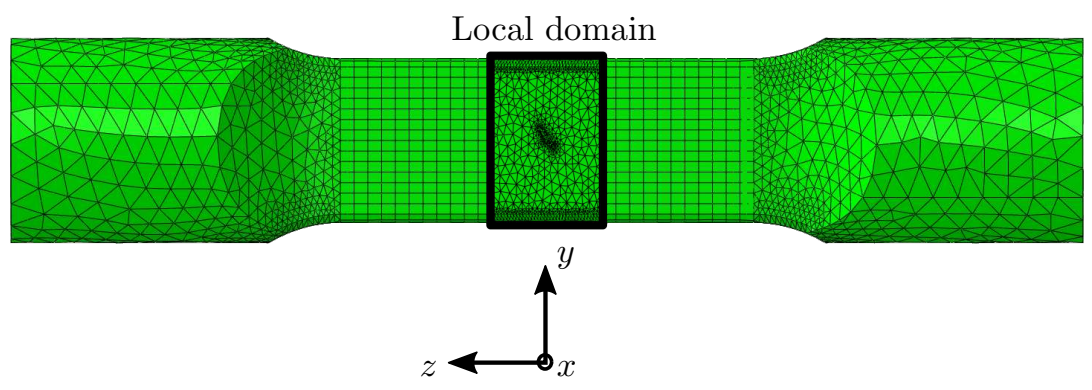

Figure 11: Mesh of the Kb specimen FE-model with the highlighted local domain and the FE coordinate system.

In order to represent the experiments in a meaningful manner the crack geometries corresponding to each of the above described crack instances were modelled. For this, the outlined crack front contours, see Fig. 5 for Kb1, were projected from the $2 \mathrm{D}$ top view on the $3 \mathrm{D}$ representation of the scanned fracture surface. The created $3 \mathrm{D}$ crack surface geometry was then transformed into a 3D triangulation and inserted in the above described local domain for further analysis. As an example, the $3 \mathrm{D}$ triangulation of the crack surface geometry of crack instance 2 in Kb1 is shown in Fig. 12 (a), where the three different crack fronts can be seen. For clarity, the crack surface geometry representation is placed at the corresponding location on the picture of the fracture surface. 


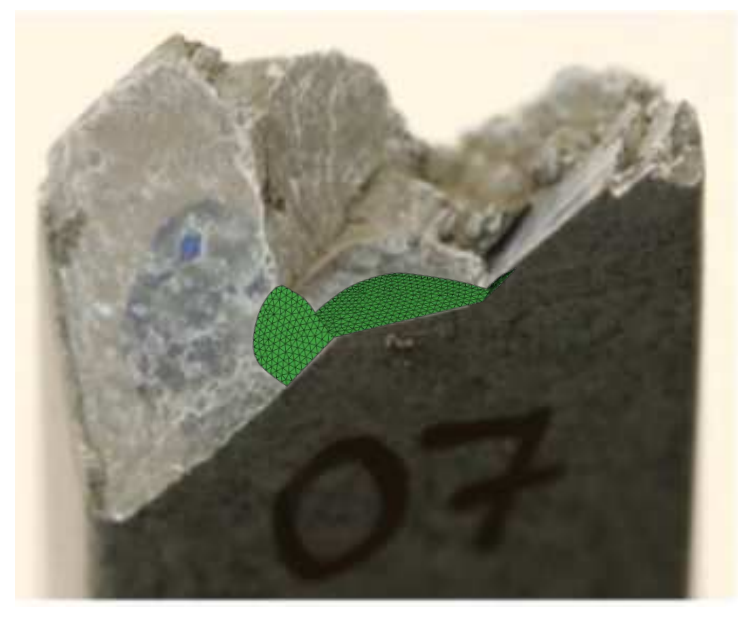

(a)

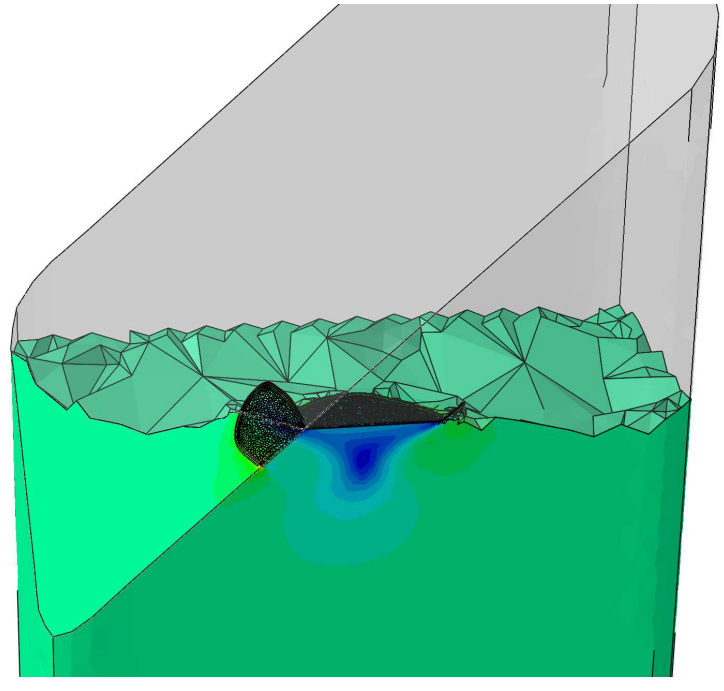

(b)

Figure 12: (a) 3D triangulation of the crack surface geometry of crack instance 2 in Kb1 overlain on the corresponding fracture surface and (b) the corresponding FE representation including the von Mises stress response with a transparent upper half of the model.

In Fig. 12 (b) the corresponding FE representation with the von Mises stress response is shown to give the reader a feeling of the complexity of the model, where the upper half of the model is transparent.

\section{Results and discussion}

The equivalent RSIFs and the crystallographic FCGR was evaluated as described above. The best results were achieved by setting the calibration parameter $\psi$ to zero. This was based on a manual maximisation of the coefficient of determination $\left(R^{2}\right)$ [31], describing the goodness of the power law fit to the crystallographic crack growth equation data, i.e. $R^{2}$ is a measure of how well the model predicts the actual experimental values. Further, it gives the percentage of variation in crystallographic crack growth rate that is accounted for by its regression on $\Delta k_{E Q}$. The closer $R^{2}$ is to unity the better is the prediction of the proposed model with respect to the experiment. The results for different 
Table 6: Comparison of $R^{2}$ for different $\psi$ values

\begin{tabular}{llllllllllll}
\hline$\psi$ & 0 & 0.1 & 0.2 & 0.3 & 0.4 & 0.5 & 0.6 & 0.7 & 0.8 & 0.9 & 1 \\
\hline$R^{2}$ & 0.79 & 0.74 & 0.68 & 0.61 & 0.54 & 0.49 & 0.44 & 0.40 & 0.37 & 0.34 & 0.31 \\
\hline
\end{tabular}

$\psi$ values ranging from zero to one are summarized in Table 6 . Based on this, it can be assumed that for crack growth on crystallographic planes, the resolved shear stresses, as in $k_{I I}$ and $k_{I I I}$, are the main driving forces, as $\psi=0$ gives the highest $R^{2}$. The resulting resolved shear stress intensity factor is then:

$$
k_{R S S}=\sqrt{k_{I I}^{2}+k_{I I I}^{2}}
$$

The physical reason for the strong dependence on the shear stresses is assumed to be that they are the main responsible for causing slip on the octahedral planes, which accumulates damage and is associated to crack growth. Similar findings have been made by Sakaguchi et al. [19], where the crystallographic crack growth rate using a crystal plasticity approach to evaluate a damage parameter based on local shear strains was studied. It was concluded that the normal stresses at the crystallographic crack fronts do not show any dominant contribution to crystallographic cracking. Nevertheless, positive resolved normal stresses are assumed to be required to ensure an open crack and to get a valid description of the crack growth. In the evaluated experiments of the present study, an open crack was assumed to be present at all times due the applied positive loading ratio of $R_{\sigma}=0.05$. It should be noted, that the contribution of the Mode I RSIF presumably depends on more factors which are not accounted for in this study as e.g. the chemical composition of single-crystal superalloy, crystallographic orientation and stress ratio.

The evaluated entities were then plotted in log-log diagram of the crystallographic FCGR against the $k_{R S S}$ parameter as described above. The results are shown in Fig. 13, where it can be seen that all data points for the crystallographic cracks follow the same trend. The circles, squares and asterisks correspond to the specimens $\mathrm{Kb} 1, \mathrm{~Kb} 2$ and $\mathrm{Kb} 3$, respectively. A power law 
fitting was used to determine the crystallographic crack growth parameters to $n_{c}=4.7541$ and $C_{c}=4.8739 \cdot 10^{-8}$ for $k_{R S S}$ in $[\mathrm{MPa} \sqrt{m}]$ and $(d L / d N)_{c}$ in $[\mathrm{mm} /$ cycle]. The corresponding trendline for the crystallographic FCGR is also shown in Fig. 13 as a solid black line. The upper and lower bounds of the scatter band were defined by applying scaling factors to the average trendline. It was found that all data points which were assumed to lie in the Paris region are confined in the scatter band by the factor of 5.1 increase and reduction from the average trendline. The scatter of the presented crystallographic FCGR results may seem high compared to other FCGR evaluations. Nevertheless, it is important to recognize that there are many different aspects contributing to the evaluation performed in this work. There are many material characteristics that are difficult to account for and can be a source of scatter in $(d L / d N)_{c}$, e.g. micro-structure or deviations in the crystallographic orientation. This can be between heat batches and between test specimens. Considering that the reproducibility of experiments of a highly homogeneous nickel alloy is typically $\pm 31 \%$ average and $\pm 50 \%$ maximum [32], the presented results, which include different loading levels, crystallographic orientations (due to the material misalignments) and partly different loading frequencies, are within satisfactory bounds for industrial purposes. The trendline was calibrated by the data points of the specimens $\mathrm{Kb} 1$ and $\mathrm{Kb} 3$. The data points for $\mathrm{Kb} 2$ were not included in the fitting. It can be seen that the Kb2 data points lie close to the trendline showing a good agreement between the calibrated trendline and the separate evaluation of $\mathrm{Kb} 2$. It can be seen that some data points for lower $k_{R S S}$ values for the right crystallographic crack of Kb1 and Kb3, cf. Kb1 and Kb3 Right in Fig. 13, lie outside the scatter band. Those data points are assumed to lie outside the Paris region. 


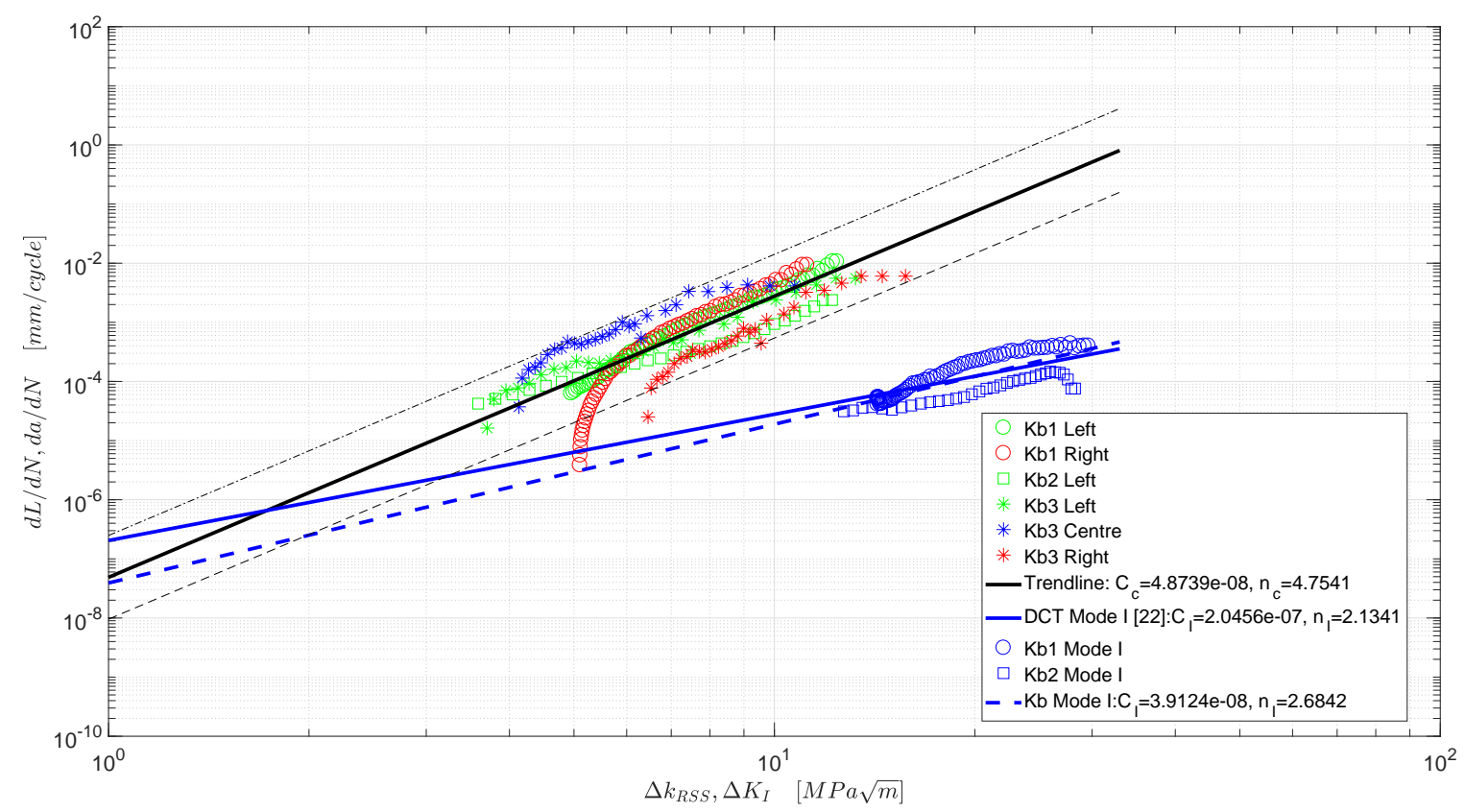

Figure 13: FCGR for crystallographic cracking with dL/dN over $k_{R S S}$ and for Mode I cracking with da/dN over $K_{I}$.

Furthermore, two trendlines of the FCGR for Mode I are given in the same plot. The Mode I crack growth parameters $C_{I}$ and $n_{I}$ for Kb Mode I as shown in Fig. 13 were evaluated as described above using the relation in Eq. 3, resulting in the dashed blue trendline. The solid blue trendline was evaluated by experiments on Disc shaped Compact Tension (DCT) specimens by Palmert et al. [22], where $K_{I}$ was evaluated according to the equations by Newman [33] that are included in ASTM E399. The DCT specimens are of the same material and tested at the same temperature. It can be seen that the Mode I trendlines for both specimen geometries lie in the same region. These trendlines are shown for informational purpose to give the reader a feeling of the Mode I FCGR and should not be compared to the crystallographic FCGR trendlines since the evaluated CDF parameters are different.

Furthermore, it should be noted that the actual crack geometries for the 
different crack instances are only partly known and, as described above, the sections hidden beneath the Mode I portion of the cracks were approximated. Thus, the stress states along the crack fronts in the experiment could deviate from the ones in the simulation. Furthermore, uncertainties in the determination of the misalignments and low-angle grain boundaries can alter the stress state and thus influence the modelling of crack driving forces. It should be noted, that the presented numerical results are obtained under the specific condition of the performed experiments. In order to apply the model to other conditions further comparisons to additional different experiments and loading conditions are needed to verify the determined parameters.

\section{Conclusions}

A methodology to evaluate the crystallographic FCGR in isothermal low-

cycle fatigue experiments at $500^{\circ} \mathrm{C}$ for a single-crystal nickel-base superalloy has been developed.

1. The data points of the crystallographic FCGR collapse within a narrow scatter band for the crystallographic cracks indicating a correlation between crystallographic crack propagation rate and the determined crystallographic CDF.

2. 3D crack growth modelling of complex crack geometries with multiple intersecting crack fronts could be achieved.

3. The parameters of the calibrated crack growth model were evaluated to be $n_{c}=4.7541$ and $C_{c}=4.8739 \cdot 10^{-8}$ for $k_{E Q}$ in $[\mathrm{MPa} \sqrt{m}]$ and $(d L / d N)_{c}$ in $[\mathrm{mm} / \mathrm{cycle}]$.

\section{Acknowledgements}

This research has been funded by Linköping University and Siemens Industrial Turbomachinery AB, the support of which is gratefully acknowledged. The authors would also like to thank Dr. Paul Wawrzynek at Fracture Analysis Consultants Inc. for all his support. 


\section{References}

[1] A. Pineau, S. D. Antolovich, High temperature fatigue of nickel-base superalloys - A review with special emphasis on deformation modes and oxidation, Engineering Failure Analysis 16 (2009) 2668-2697.

[2] D. Leidermark, J. Moverare, K. Simonsson, S. Sjöström, S. Johansson, Room temperature yield behaviour of a single-crystal nickel-base superalloy with tension/compression asymmetry, Computational Materials Science 47 (2009) 366-372.

[3] D. P. Pope, S. S. Ezz, Mechanical properties of Ni3Al and nickel-base alloys with high volume fraction of gamma prime, International metals reviews 29 (1984) 136-167.

[4] M. Segersäll, J. Moverare, D. Leidermark, K. Simonsson, Low-cycle fatigue behaviour of a Ni-based single-crystal superalloy, volume 891-892, 2014. doi:10.4028/www.scientific.net/AMR.891-892.416.

[5] D. Leidermark, M. Segersäll, Modelling of thermomechanical fatigue stress relaxation in a single-crystal nickel-base superalloy, Computational Materials Science 90 (2014) 61-70.

[6] R. L. Amaro, S. D. Antolovich, R. W. Neu, A. Staroselsky, Physics-Based Modeling of Thermo-Mechanical Fatigue in PWA 1484, in: Superalloys 2012, 2012, pp. 481-490. doi:10.1002/9781118516430.ch53.

[7] B. F. Antolovich, A. Saxena, S. D. Antolovich, Fatigue crack propagation in single-crystal CMSX- 2 at elevated temperature, Journal of Materials Engineering and Performance 2 (1993) 489-495.

[8] J. Telesman, L. J. Ghosn, The unusual near-threshold FCG behavior of a single crystal superalloy and the resolved shear stress as the crack driving force, Engineering Fracture Mechanics 34 (1989) 1183-1196. 
[9] M. Sakaguchi, T. Tsuru, M. Okazaki, Fatigue Crack Propagation in Thin-Wall Superalloys Component; Experimental Investigation via Miniature CT Specimen, in: Superalloys 2012, 2012. doi:10.1002/9781118516430.ch47.

[10] S. Suzuki, M. Sakaguchi, H. Inoue, Temperature dependent fatigue crack propagation in a single crystal Ni-base superalloy affected by primary and secondary orientations, Materials Science and Engineering A (2018).

[11] H. Kagawa, Y. Mukai, The Effect of Crystal Orientation and Temperature on Fatigue Crack Growth of Ni-Based Single Crystal Superalloy, John Wiley and Sons, 2012, pp. 225-233. doi:10.1002/9781118516430.ch25.

[12] J. Telesman, L. J. Ghosn, Fatigue crack growth behavior of PWA 1484 single crystal superalloy at elevated temperatures, Journal of Engineering for Gas Turbines and Power 118 (1996) 399-405.

[13] D. Leidermark, J. Moverare, K. Simonsson, S. Sjöström, A combined critical plane and critical distance approach for predicting fatigue crack initiation in notched single-crystal superalloy components, International Journal of Fatigue 33 (2011) 1351-1359.

[14] T. Tinga, Stress intensity factors and crack propagation in a single crystal nickel-based superalloy, Engineering Fracture Mechanics 73 (2006) 16791692.

[15] K. S. Chan, J. Feiger, Y. D. Lee, R. John, S. J. Hudak, Fatigue crack growth thresholds of deflected mixed-mode cracks in PWA1484, volume 127, 2005, pp. 2-7. doi:10.1115/1.1836765.

[16] R. W. Neu, Crack paths in single-crystal Ni-base superalloys under isothermal and thermomechanical fatigue, International Journal of Fatigue 123 (2019) 268-278. 
[17] D. MacLachlan, D. Knowles, Fatigue behaviour and lifing of two single crystal superalloys, Fatigue and Fracture of Engineering Materials and Structures 24 (2001).

[18] C. Busse, J. Homs, D. Gustafsson, F. Palmert, B. Sjödin, J. Moverare, K. Simonsson, D. Leidermark, A finite element study of the effect of crystal orientation and misalignment on the crack driving force in a single-crystal superalloy, in: Proceedings of the ASME Turbo Expo, volume 7A-2016, 2016. doi:10.1115/GT2016-56305.

[19] M. Sakaguchi, R. Komamura, X. Chen, M. Higaki, H. Inoue, Crystal plasticity assessment of crystallographic Stage I crack propagation in a Ni-based single crystal superalloy, International Journal of Fatigue (2019).

[20] H. W. Liu, Q. Chen, D. Lai, Shear fatigue crack growth and its analysis 29 (1993) 300-307.

[21] Q. Chen, H. Liu, Resolved shear stress intensity coefficient and fatigue crack growth in large crystals, Theoretical and Applied Fracture Mechanics 10 (1988) 111-122.

[22] F. Palmert, J. Moverare, D. Gustafsson, C. Busse, Fatigue crack growth behaviour of an alternative single crystal nickel base superalloy, International Journal of Fatigue 109 (2018) 166-181.

[23] R. Reed, J. Moverare, A. Sato, F. Karlsson, M. Hasselqvist, A New Single Crystal Superalloy for Power Generation Applications, Superalloys 2012 (2012) 197-204.

[24] A. Coles, R. E. Johnson, H. G. Popp, Utility of surface-flawed tensile bars in cyclic life studies, Journal of Engineering Materials and Technology, Transactions of the ASME 98 (1976) 305-315.

[25] C. Busse, F. Palmert, B. Sjödin, P. Almroth, D. Gustafsson, K. Simonsson, D. Leidermark, Prediction of crystallographic cracking planes in single- 
crystal nickel-base superalloys, Engineering Fracture Mechanics 196 (2018) 206-223.

[26] D. R. Askeland, P. P. Phule, The Science and Engineering of Materials, fifth edit ed., Thomson, Canada, Toronto, 2006.

[27] L. Banks-Sills, P. A. Wawrzynek, B. Carter, A. R. Ingraffea, I. Hershkovitz, Methods for calculating stress intensity factors in anisotropic materials: Part II-Arbitrary geometry, Engineering Fracture Mechanics 74 (2007) $1293-1307$.

[28] P. Paris, F. Erdogan, A Critical Analysis of Crack Propagation Laws, Journal of Basic Engineering 85 (1963) 528-533.

[29] FRANC3D, FRANC3D Reference Manual, Fracture Analysis Consultants Inc., Ithaca, USA, 2016.

[30] ABAQUS, ABAQUS 6.12 Documentation, Dassault Systèmes, Providence, USA, 2014.

[31] N. R. Draper, H. Smith, Applied Regression Analysis, 3rd ed., WileyInterscience, 1998. doi:10.1002/9781118625590.

[32] ASTM E647-13, Standard Test Method for Measurement of Fatigue Crack Growth Rates, American Society for Testing and Materials (2014).

[33] J. C. Newman, Stress-intensity factors and crack-opening displacements for round compact specimens, International Journal of Fracture (1981). 